\begin{tabular}{|c|l|}
\hline Title & Distinctive stability of a free standing monolayer clay mineral nanosheet via transmission electron microscopy \\
\hline Author(s) & A kita, Ikumi; Ishida, Y ohei; Y onezawa, Tetsu \\
\hline Citation & $\begin{array}{l}\text { Physical chemistry chemical physics, 22(43), 25095-25102 } \\
\text { https://doi.org/L0.1039/10cp04659k }\end{array}$ \\
\hline Issue Date & $2020-11$-21 \\
\hline Doc URL & http://hdl.handle.net/2115/83303 \\
\hline Type & article (author version) \\
\hline Additional Information & There are other files related to this item in HUSCAP. Check the above URL. \\
\hline File Information & Manuscript (Ishida).pdf \\
\hline
\end{tabular}

Instructions for use 


\section{Distinctive Stability of Free-standing Monolayer Clay Mineral Nanosheet under Transmission Electron Microscopy}

Received 00th January 20xx, Accepted 00th January 20xx DOI: $10.1039 / \times 0 \times x 00000 x$

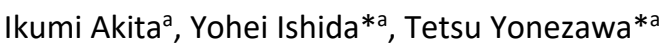

Among 2D materials, clay mineral nanosheets have been extensively studied owing to their specific features, such as high surface charge and large surface area. Recently, we reported a stable free-standing (without any surfactants or matrices) monolayer clay mineral, characterized via annular dark-field scanning transmission electron microscopy (ADF-STEM) at the atomic-scale. Herein, we demonstrated that the monolayer clay mineral exhibited the outstanding stability under electron beam irradiation compared to two- or three-layered nanosheets via electron diffraction analysis. In addition to its low thickness ( $1 \mathrm{~nm}$-thick), the absence of an interlayer space was the critical factor contributed to the distinctive stability of the monolayer clay mineral, compared to that of the two- or three-layered clay mineral. The findings here inspire further investigation in free-standing clay mineral using (S)TEM and also for a wide variety of nanomaterials which are strongly hydrated.
\end{abstract}

\section{Introduction}

Clay minerals are phyllosilicates, featuring physicochemical properties such as atomically flat surfaces with a large surface area, high surface charges, and high thermal stability. Utilizing these features, clay minerals have been used in various fields ranging from environmental applications (as absorbents, ion exchangers, and water decontamination) to fine chemical synthesis. ${ }^{1-3}$ Among the many types of clay minerals, smectite groups (e.g., saponite, montmorillonite) are frequently used owing to their moderate anionic charge density, imparting exfoliation (or stacking) ability to individual nanosheets in aqueous solutions as well as optical transparency in the visible light region in the exfoliated state at small particle sizes. ${ }^{4-7}$ Smectite possess the following chemical formula: $\left[\left(\mathrm{Si}_{8-\mathrm{x}} \mathrm{Al}_{\mathrm{x}}\right)\left(\mathrm{Mg}_{6}\right.\right.$ $\left.\left.{ }_{y} \mathrm{Al}_{\mathrm{y}}\right) \mathrm{O}_{20}(\mathrm{OH})_{4}\right]^{\cdot}(\mathrm{x}-\mathrm{y}) \mathrm{Y}^{+}$, where $\mathrm{Y}$ represents the cations to balance the charges, and they possess layers consisting of a 2:1 ratio of tetrahedral and octahedral sheets, with a thickness of $0.96 \mathrm{~nm}$. Completely exfoliated clay mineral, as a single layer, can be defined as a family of two-dimensional (2D) nanosheets.

Recently, characterization of 2D nanomaterials has greatly advanced owing to the atomic-scale imaging using aberrationcorrected transmission electron microscopy (TEM) or scanning transmission electron microscopy (STEM). ${ }^{8-13}$ Furthermore, for materials with simple structures such as graphene and transition metal dichalcogenide nanosheets, single-atom and single-molecule observations within hybrid materials have been

\footnotetext{
a. Division of Materials Science and Engineering, Faculty of Engineering, Hokkaido University, Kita 13 Nishi 8, Kita-ku, Sapporo, Hokkaido 060-8628, Japan *Corresponding Authors Email: ishida-yohei@eng.hokudai.ac.jp; tetsu@eng.hokudai.ac.jp

+Electronic Supplementary Information (ESI) available: Additional relative SAED intensity decay for $2 L \mathrm{Mt}$, and SAED patterns at difference dose rate. See DOI: $10.1039 / x 0 \times x 00000 x$
}

achieved. ${ }^{14-21}$ However, (S)TEM imaging of free-standing monolayer clay minerals, which have a more complicated structure than their preceding few-atom thick materials, such as graphene, at the atomic-scale has not been achieved, except in our recent study. ${ }^{22}$ Once non-destructive direct imaging of clay mineral nanosheet or useful clay mineral-based organicinorganic hybrid materials at the atomic scale is established, it will strongly promote their applicability such as selective adsorption for heterogeneous catalyst or sensing, ${ }^{23}$ or photoenergy conversion. ${ }^{5}$

We recently achieved atomic-scale imaging of a freestanding (i.e., without any surfactants or supporting matrices) monolayer clay mineral by annular dark-field (ADF) STEM. ${ }^{22,24}$ The monolayer clay mineral was observed at the atomic-scale without any destruction, although bulk clay minerals are generally sensitive to the electron beam irradiation, as reported for cross-sectional imaging at the atomic-scale via (S)TEM. ${ }^{25-29}$ In this report, we demonstrate the outstanding stability of freestanding monolayer clay mineral by comparing the decrease of selected area electron diffraction (SAED) intensity against those of the two- or three-layered ( $2 \mathrm{~L}$ or $3 \mathrm{~L}$ ) clay mineral. The critical dose was determined as the point at which the electron diffraction intensities were reduced to $1 /$ e (corresponding to $37 \%$ reduction) of its initial value. In addition to the fascinating property of clay mineral as itself, clay mineral nanosheets can serve as a simple model for considering beam-damage mechanisms in oxide nanosheets without using any surfactants that are commonly required for many $2 \mathrm{D}$ materials in their synthetic or exfoliation processes. ${ }^{8,30}$ The results here revealed the crucial effect of interlayer spaces to the beam damage mechanism rather than the thickness among a few layers of clay mineral, which can also be useful for understanding the electron beam damage to a wide variety of nanomaterials that are strongly hydrated. 


\section{Methods}

Sample preparation. Montmorillonite (Mt), a typical anionic clay mineral, was acquired from Kunimine Industries (Kunipia $F$ with the chemical structure corresponding to $\mathrm{Na}_{0.35} \mathrm{~K}_{0.01} \mathrm{Ca}_{0.06}\left(\mathrm{Al}_{1.56} \mathrm{Mg}_{0.33} \mathrm{Fe}_{0.09} \mathrm{Ti}_{0.01}\right)\left(\mathrm{Si}_{3.87} \mathrm{Al}_{0.13}\right) \mathrm{O}_{10}(\mathrm{OH})_{2}$ and the cation exchange capacity of 1.19 meq. $\mathrm{g}^{-1}$ ) ${ }^{31,32}$ and was purified using the following procedure. First, raw clay mineral dispersion ( $300 \mathrm{~mL}, 10 \mathrm{~g} \mathrm{~L}^{-1}$ ) was placed in centrifuge bottles, which were then centrifuged at a rotation speed of $7000 \mathrm{rpm}$ for $15 \mathrm{~min}$. The obtained supernatant was collected and poured into a container with $1500 \mathrm{~mL}$ of ethanol. After $1 \mathrm{~h}$ of mixing at a temperature of $70{ }^{\circ} \mathrm{C}$, the resulting colloidal solution was filtered using a PTFE membrane with a pore size of $0.1 \mu \mathrm{m}$ (Millipore). The produced cake was collected and dried under vacuum by rotary pump overnight. The dried cake was dispersed in water, and the dispersion was stored overnight to achieve complete exfoliation into nanosheets. A specimen for STEM/TEM was prepared by drop casting the aqueous solution of monolayer Mt on a holey carbon-coated Cu grid.

ADF-STEM observation. For the STEM observations for determination of the layer number, a Titan Cubed $G 2$ equipped with spherical aberration correctors was used at an acceleration voltage of $300 \mathrm{kV}$. The convergence semi-angle of the incident probe was $21.4 \mathrm{mrad}$. The inner and outer detection semi-angle of ADF detector were 31.4 and 192 mrad, respectively. The incident probe current was set to $\sim 30 \mathrm{pA}$.

Time series of TEM-SAED. TEM-SAED observation was performed by using the Titan Cubed $\mathrm{G} 2$ at an acceleration voltage of $300 \mathrm{kV}$. The dose rate was set to $10 \mathrm{e}^{-} \AA^{-2} \mathrm{~s}^{-1}$ by monitoring the screen current and the exposure time was $3 \mathrm{~s}$ for each image acquisition. Note that there is no calibration from the screen current to the actual number of electrons.

\section{Results and Discussion}

\section{Determination of the stacking number of free-standing Mt nanosheet by ADF-STEM observation.}

The atomic structure of the unit layer structure for typical monolayer montmorillonite (1L Mt) is shown in Fig. 1a. The unit layer comprises 2:1 combination of tetrahedral silica and octahedral aluminum hydroxide sheets. The general chemical formula of the layered aluminosilicates, such as $\mathrm{Mt}$, mica, and pyrophyllite, is $\left[\mathrm{Si}_{4} \mathrm{O}_{8}\right]\left[\mathrm{Al}_{2} \mathrm{O}_{2}(\mathrm{OH})_{2}\right]$. Two-thirds of the oxygen atoms in the octahedral sheet are supplied by silica tetrahedra, and the others are supplied by hydroxyl groups (indicated as yellow spheres in Fig. 1a). The isomorphous substitution of $\mathrm{Al}^{3+}$ to $\mathrm{Mg}^{2+}$ in the octahedral sheet produces anionic charges within the $\mathrm{Mt}$ structure. Fig. $1 \mathrm{~b}$ shows the ADF-STEM image of the free-standing $\mathrm{Mt}$ nanosheets on a holey carbon film at low magnification. The acceleration voltage and the incident probe current were $300 \mathrm{kV}$ and $\sim 30 \mathrm{pA}$, respectively. The free-standing Mt sample was prepared by pouring an aqueous dispersion of the exfoliated monolayer Mt onto a holey carbon- coated $\mathrm{Cu}$ grid. As shown in Fig. 1b, the free- standing Mt nanosheets were
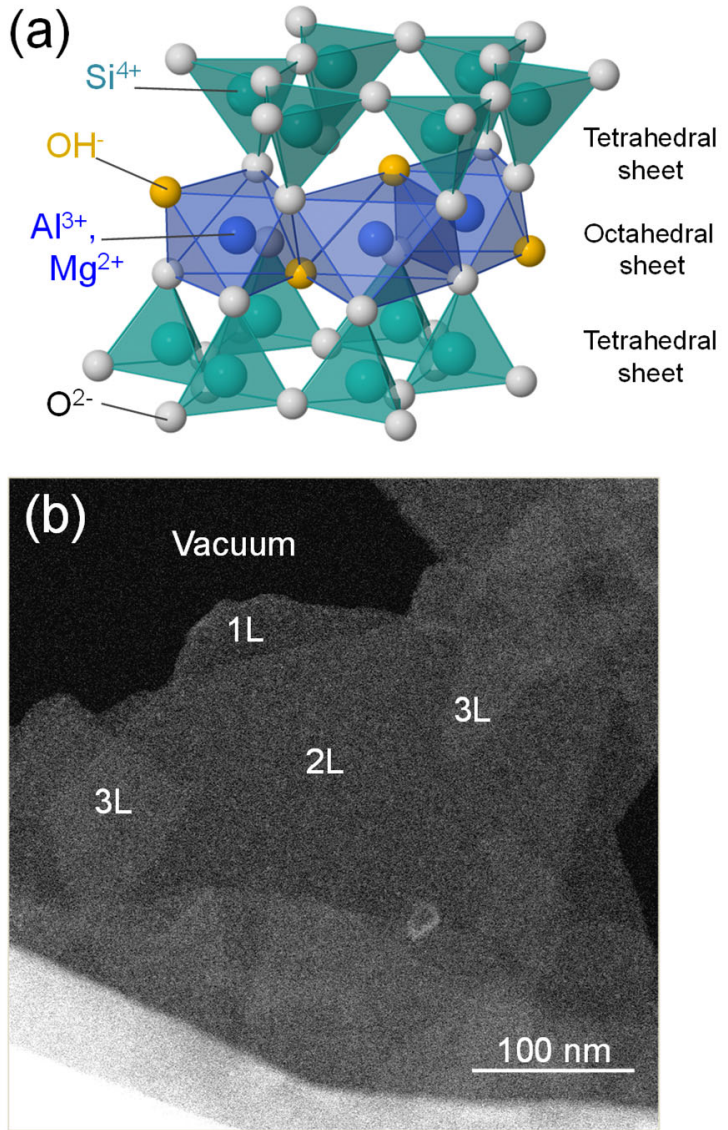

Fig. 1. (a) Atomic structure model for a unit layer of montmorillonite showing the octahedral alumina sheet sandwiched by two tetrahedral silica sheets. Hydrogen atoms at the tetrahedral sheet edge are omitted for clarity. (b) ADF-STEM image of free-standing Mt nanosheets deposited on a holey carbon grid, and the corresponding stacked-layer number from one (1L) to three $(3 \mathrm{~L})$.

stacked horizontally. Hence, the electron beam was irradiated in a direction normal to the surface. The stacking number of the $\mathrm{Mt}$ nanosheets, ranging from $1 \mathrm{~L}$ to $3 \mathrm{~L}$, was determined by the difference between their contrasts, as previously reported (Fig. 1b). ${ }^{24}$ To further analyze the stability of free-standing Mt under electron beam irradiation, we determined the critical doses of $1 \mathrm{~L}, 2 \mathrm{~L}$, and $3 \mathrm{~L} \mathrm{Mt}$ from the intensity reduction in their SAED patterns. Note that free-standing $\mathrm{Mt}$, especially $1 \mathrm{~L} \mathrm{Mt}$, were difficult to find in normal TEM, due to the ultrathin structure consisted with light elements. Therefore, the stacking number of $\mathrm{Mt}$ was determined via ADF-STEM, where the contrast positively correlated with the atomic number or thickness of the specimen. ${ }^{24}$

\section{The critical dose of electron beam damage as a function of the layer} number.

Fig. 2 shows a series of SAED patterns for (a) 1L, (b) 2L, and (c) 3L $\mathrm{Mt}$ at initial exposure and cumulative electron doses of 3,15 , and 30 $\times 103 \mathrm{e}^{-} \AA^{-2}$. The acceleration voltage and the dwell for each pattern were $300 \mathrm{kV}$ and $3 \mathrm{~s}$, respectively. For the $1 \mathrm{~L} \mathrm{Mt}$ shown in Fig. 2a, 

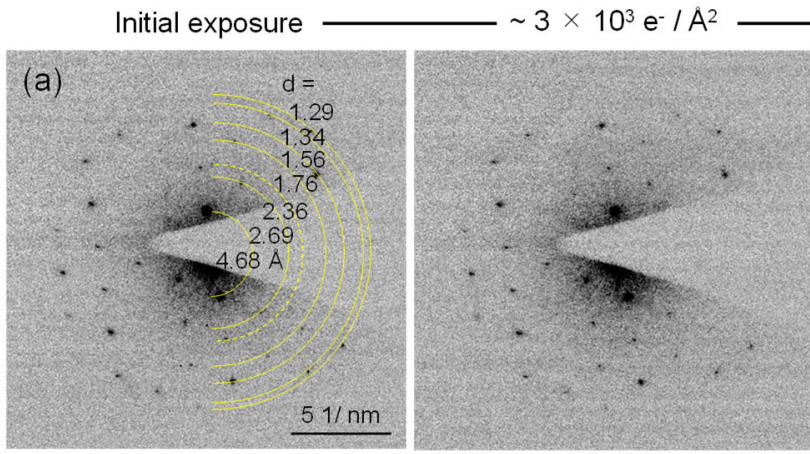
$\sim 15 \times 10^{3} \mathrm{e}^{-/} \mathrm{A}^{2}$

$\sim 30 \times 10^{3} \mathrm{e}^{-} / \mathrm{A}^{2}$
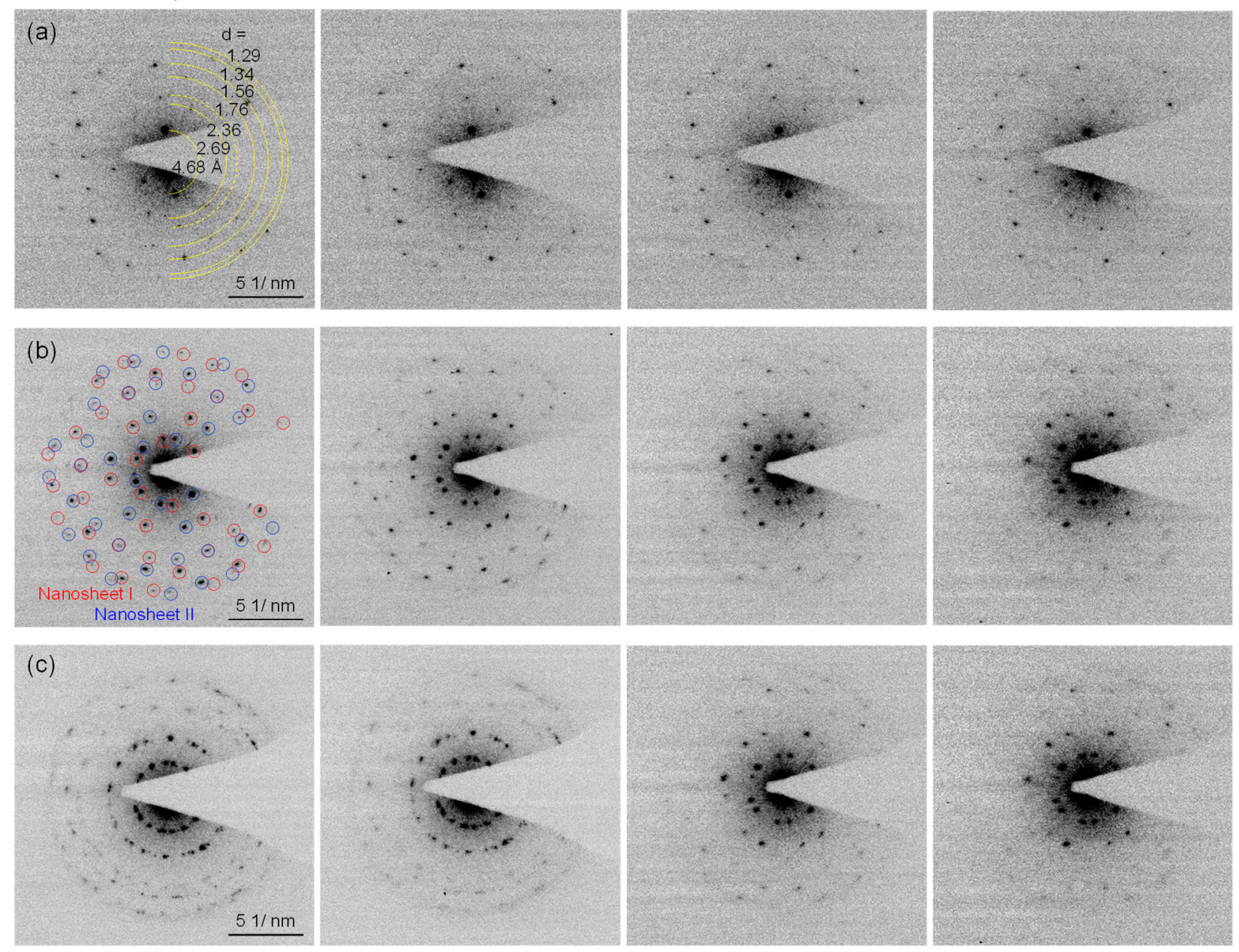

Fig. 2. Series of SAED patterns for (a) monolayer (1L), (b) two-layered (2L), and (c) three-layered (3L) Mt at initial exposure and cumulative electron doses of 3,15 , and $30 \times 10^{3} \mathrm{e}^{-} \AA^{-2}$. The acceleration voltage and the electron dose rate were $300 \mathrm{kV}$ and $10 \mathrm{e}^{-}$ $\AA^{-2} \mathrm{~s}^{-1}$, respectively. The dwell time for each pattern was set at $3 \mathrm{~s}$. The in-plane distance (d) of $1 \mathrm{~L} \mathrm{Mt}$ was indicated by yellow lines in (a). Red and blue circles in panel $b$ (left) denote diffraction from nanosheet I and II. Note that all the SAED patterns in the same line $(a, b$, and $c)$ are scaled to the same display value range.

the diffraction spots remained clearly visible even after significant exposure to electrons, approximately $30 \times 10^{3} \mathrm{e}^{-} \AA^{-2}$. The in-plane distance (d) of $1 \mathrm{~L} \mathrm{Mt} \mathrm{was} \mathrm{indicated} \mathrm{by} \mathrm{yellow} \mathrm{lines.}$ A yellow dashed line, corresponding to the d value of $2.36 \AA$, was observed only for $1 \mathrm{~L} \mathrm{Mt}$. The $d$ values summarized in Table 1 are in good agreement with the values of $(h k 0)$ facets reported elsewhere. ${ }^{33-35}$ Fig. $2 b$ and $c$ show series of SAED images for $2 \mathrm{~L}$ and $3 \mathrm{~L} \mathrm{Mt}$ at initial exposure and cumulative electron doses of 3,15 , and $30 \times 10^{3} \mathrm{e}^{-} \AA^{-2}$. The red and blue circles in Fig. $2 \mathrm{~b}$ denote diffraction from nanosheet I and II which was distinguished by comparing the pattern of $1 \mathrm{~L} \mathrm{Mt} \mathrm{nanosheet.}$ Some of the spots corresponding to the $d$ value of $1.76 \AA$ in the two nanosheets were overlapped, and they were excluded from the intensity analysis discussed later. In contrast to the results of $1 \mathrm{~L} \mathrm{Mt}$, the diffraction patterns of the $2 \mathrm{~L}$ and $3 \mathrm{~L} \mathrm{Mt}$ nanosheets show an obvious change after a certain amount of exposure, from appearing as sharp spots to blurred rings, indicating the loss of their crystallinity.
Table 1. The in-plane distance (d) of $1 \mathrm{~L} \mathrm{Mt} \mathrm{determined} \mathrm{by} \mathrm{SAED}$ at initial exposure (Fig. 2a, left). The $d$ values are in good agreement with the values of $(h k 0)$ facets reported previously..$^{30}$

\begin{tabular}{cc}
\hline \hline$(h k 0)$ & $d / \AA$ \\
\hline 11,02 & 4.68 \\
13,20 & 2.69 \\
22,04 & 2.36 \\
$31,15,24$ & 1.76 \\
33,06 & 1.56 \\
26,40 & 1.34 \\
$35,17,42$ & 1.29 \\
\hline \hline
\end{tabular}


(a)

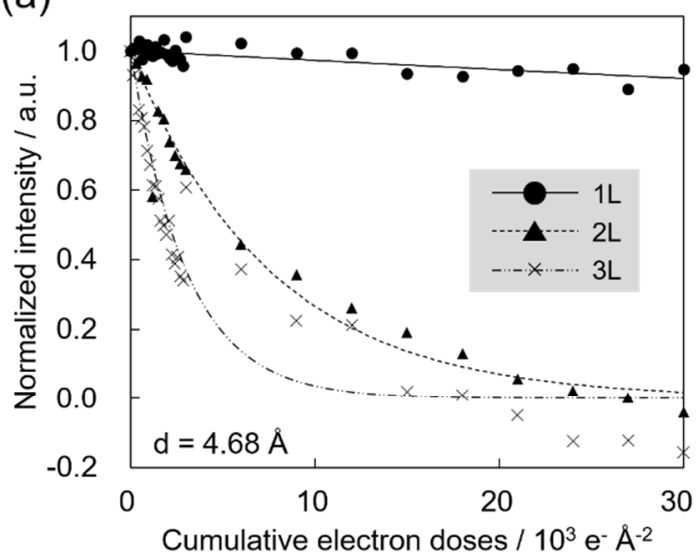

(c)

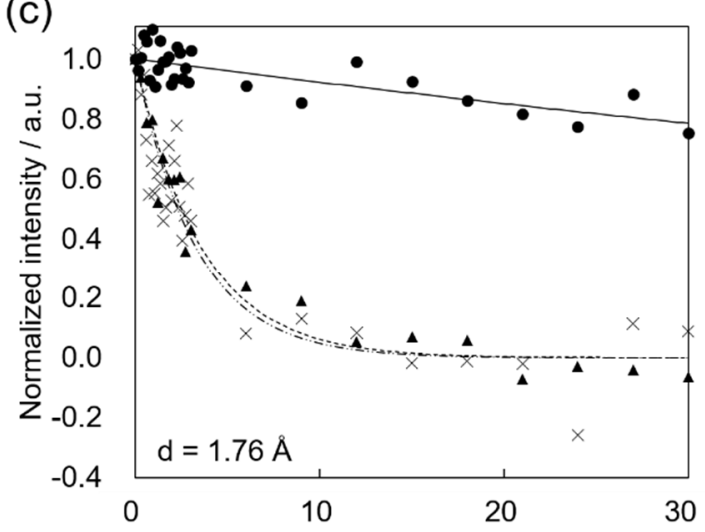

(e) Cumulative electron doses $/ 10^{3} \mathrm{e}^{-} \AA^{-2}$

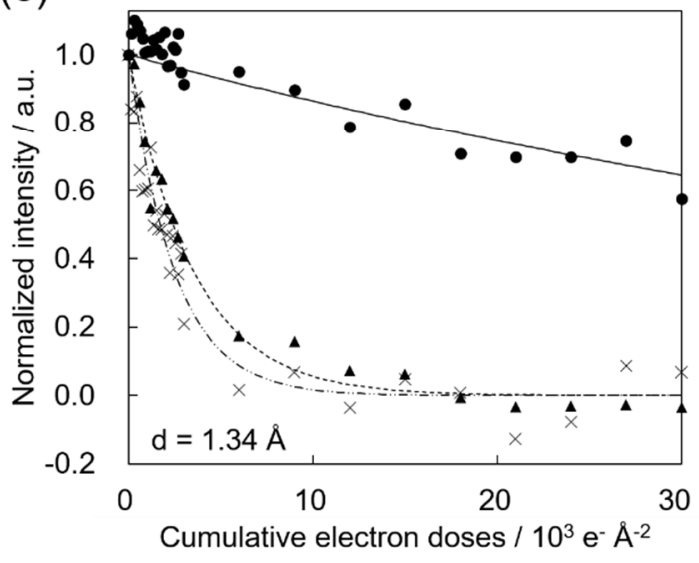

(b)

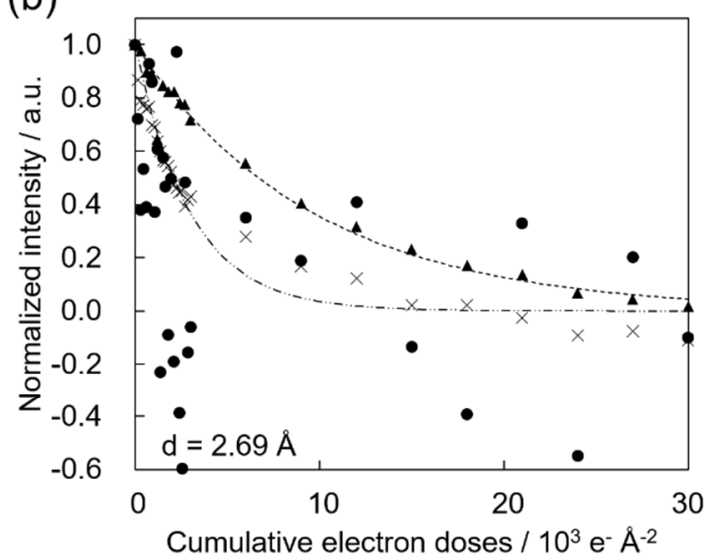

(d)

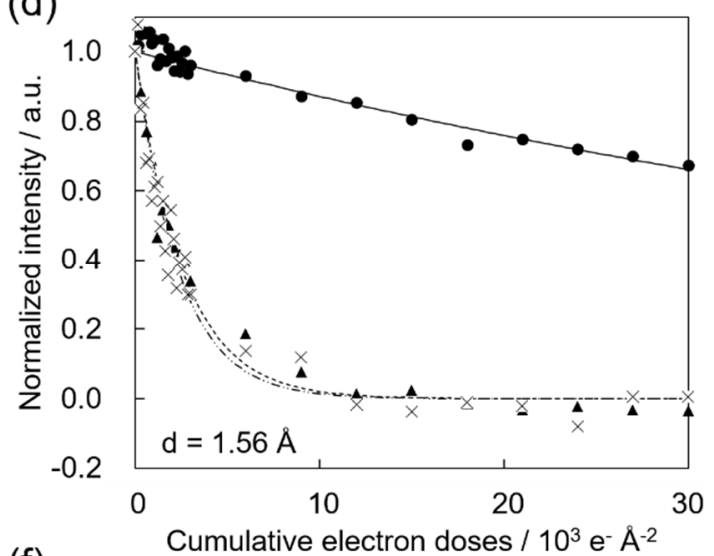

(f)

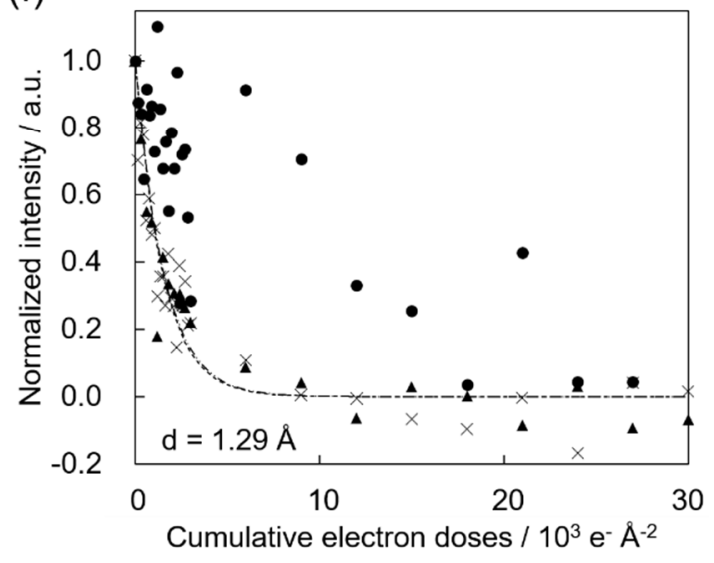

Fig. 3. Relative intensity decay for the d-spacing of (a) 4.68 , (b) 2.69 , (c) 1.76 , (d) 1.56 , (e) 1.34 , (f) $1.29 \AA$, corresponding to the diffraction from $(11,02) ;(13,20) ;(31,15,24) ;(33,06) ;(26,40)$; and $(35,17,42)$ facets as a function of the cumulative electron doses for $1 \mathrm{~L}(\bullet), 2 \mathrm{~L}(\mathbf{\Delta})$, and $3 \mathrm{~L} \mathrm{Mt}(\times)$, respectively. The acceleration voltage and the electron dose rate were $300 \mathrm{kV}$ and $30 \times 10^{3}$ $\mathrm{e}^{-} \AA^{-2} \mathrm{~s}^{-1}$, respectively.

The outer spots, corresponding to smaller atomic spacing such as 1.34 or $1.29 \AA$, reduced rapidly, indicating their sensitivity to the electron beam irradiation.

Fig. 3 shows normalized intensity decay for the d-spacing of (a) 4.68 , (b) 2.69 , (c) 1.76 , (d) 1.56 , (e) 1.34 , (f) $1.29 \AA$, corresponding to the diffraction from $(11,02) ;(13,20) ;(31,15$, $24) ;(33,06) ;(26,40)$; and $(35,17,42)$ facets as a function of the cumulative electron doses for $1 \mathrm{~L}(\bullet), 2 \mathrm{~L}(\boldsymbol{\Delta})$, 3L Mt $(\times)$, respectively. Black lines in Fig. 3 indicate exponential decay curves determined by the least-squares method. The critical doses for each curve, determined as the point at which the electron diffraction intensities reduced to $1 /$ e (corresponding to $37 \%$ reduction) of their initial value, are summarized in Table 2. Fig. 3 was normalized to compare the critical doses of different stacked number of $\mathrm{Mt}$ nanosheets. An exponential approximation was first performed and the obtained constant 
Table 2. The critical doses for $1 \mathrm{~L}, 2 \mathrm{~L}, 3 \mathrm{~L} \mathrm{Mt}$, determined by exponential approximation in Fig. 3.

\begin{tabular}{ccccc}
\hline \hline & & \multicolumn{3}{c}{ Critical dose $\left(10^{3} \mathrm{e}^{-} \AA^{-2}\right)$} \\
\cline { 3 - 5 } & & $1 \mathrm{~L}$ & $2 \mathrm{~L}$ & $3 \mathrm{~L}$ \\
\hline (a) & 4.68 & 363.02 & 7.55 & 3.02 \\
(b) & 2.69 & - & 9.64 & 2.99 \\
(c) & 1.76 & 124.36 & 4.08 & 3.31 \\
(d) & 1.56 & 72.60 & 2.58 & 2.35 \\
(e) & 1.34 & 68.29 & 3.48 & 2.46 \\
(f) & 1.29 & - & 1.49 & 1.54 \\
\hline \hline
\end{tabular}

Table 3. The critical doses for the two component nanosheets (noted as nanosheet I and II) in $2 \mathrm{~L} \mathrm{Mt}$ as indicated by red and blue circles in Fig. 2b. The values were determined by exponential approximation in Fig. S1.

\begin{tabular}{ccc}
\hline \hline & \multicolumn{2}{c}{ Critical dose $/\left(10^{3} \mathrm{e}^{-} \AA^{-2}\right)$} \\
\cline { 2 - 3 } & Nanosheet I & Nanosheet II \\
\hline 4.68 & 11.14 & 7.02 \\
2.69 & 24.35 & 6.61 \\
1.76 & 2.51 & 5.16 \\
1.56 & 2.44 & 1.97 \\
1.34 & 3.29 & 3.9 \\
1.29 & 1.79 & 1.07 \\
\hline \hline
\end{tabular}

term was set to zero, the data set was then normalized. Some negative values were found for the data set with a large variation relative to the absolute intensities. exponential approximation was first performed and the obtained constant term was set to zero, the data set was then normalized. Some negative values were found for the data set with a large variation relative to the absolute intensities. Note that normalization and exponential curve fitting for the d-spacing of 2.69 and $1.29 \AA$ of $1 \mathrm{~L} \mathrm{Mt}$ was not performed correctly due to the large variation of the intensity. All critical doses for $1 \mathrm{~L} \mathrm{Mt}$ were significantly larger than those for $2 \mathrm{~L}$ or $3 \mathrm{~L} \mathrm{Mt}$ ones. The critical dose for $2 \mathrm{~L} \mathrm{Mt}$, however, was in the same order as that of $3 \mathrm{~L} \mathrm{Mt}$. According to the results of $1 \mathrm{~L} \mathrm{Mt}$ the outer spots corresponding to smaller atomic spacing were reduced rapidly, indicating their sensitivity to the electron beam irradiation. For $2 \mathrm{~L}$ and $3 \mathrm{~L} \mathrm{Mt}$, the relationship between the critical dose and the $\mathrm{d}$-spacing value was no longer observable. Additionally, Table 3 shows the critical doses for the two component nanosheets (noted as nanosheet I and II) in $2 \mathrm{~L} \mathrm{Mt}$ as indicated by red and blue circles in Fig. 2b. Since there was no significant difference in the critical dose of nanosheets I and II, the ordering between nanosheets I and II against the incident direction of electron beam could not be determined from SAED analysis (See Fig. S1 in the Supplementary Information for the entire decay curves). These results clearly demonstrate that the free-standing $1 \mathrm{~L} \mathrm{Mt}$ nanosheet showed unexpected, distinct stability compared with $2 \mathrm{~L}$ or more layered $\mathrm{Mt}$ under electron beam irradiation.

\section{Mechanism of electron beam damage on $\mathrm{Mt}$ and the role of the interlayer space.}

In free-standing Mt nanosheets during the TEM observation, multiple electron beam damage mechanisms can proceed simultaneously. The dominant mechanism of beam damage in insulating materials such as clay minerals is radiolysis damage caused by inelastic scattering - the transfer of energy from the incident electron to the electrons in the specimen. Other mechanism includes local heating or knock-on damage. The local heating also proceed the damage rate due to low thermal conductivity of clay minerals. Additionally, any hydrogens in the sample readily stripped by electron beam irradiation at the acceleration voltage of $300 \mathrm{kV}$, and the emitted hydrogens with high kinetic energy can cause knock-on damage in the structure.

Recently we demonstrated the stable ADF-STEM imaging of free-standing monolayer $\mathrm{Mt}$ at the atomic scale, and concluded that radiolysis damage were effectively suppressed due to the thin structure of the $1 \mathrm{~L} \mathrm{Mt}(0.96 \mathrm{~nm})$ compared to the mean free path of secondary electrons in the oxides (ca. 10-20 nm). ${ }^{36-}$ ${ }^{38}$ Radiolysis damage is caused by secondary electrons produced by inelastic scattering, and the possibility of secondary electrons escaping from the sample surface into the vacuum depends on their depth from the surface. ${ }^{36}$ Therefore, if the sample is an atomically thin film or small particulate matter, the beam sensitivity decreases as the secondary electrons excited from near-surface atoms escape from the specimen to vacuum without causing damage. ${ }^{37-39}$ The suppression of electron damage by lowering the dimension of the sample (from 3D to 2D or 1D) is becoming commonplace for direct imaging of beam-sensitive materials by electron microscopy. ${ }^{15,39}$

Note that since the escaped secondary electrons are not compensated by the neighboring electrons quickly in insulator, the specimen can be positively charged. For ionic materials such as clay minerals, this positive charge can induce an electric field in the specimen, which results in image drift or distortion, structural change, mass transport, or Coulomb explosion in some case. To avoid such explosive damage, we optimized the dose rate as $10 \mathrm{e}^{-} \AA^{-2} \mathrm{~s}^{-1}$, which is mild condition that allows us to track the destruction process by SAED pattern. At high dose rate we could not get the fine SAED pattern even at the first scanning, which indicating the specimen was already destructed (See Fig. S2 in the Supplementary Information). Such drastic destruction might be caused by Coulomb explosion at high dose rate.

In the present report, however, only the $1 \mathrm{~L} \mathrm{Mt}$ showed the remarkably high stability against to the electron beam irradiation, despite $2 \mathrm{~L}$ and $3 \mathrm{~L}$ samples being thin in comparison with the mean free path of secondary electrons. ${ }^{36-38}$ Also, the 
(a) 1L-Mt nanosheet

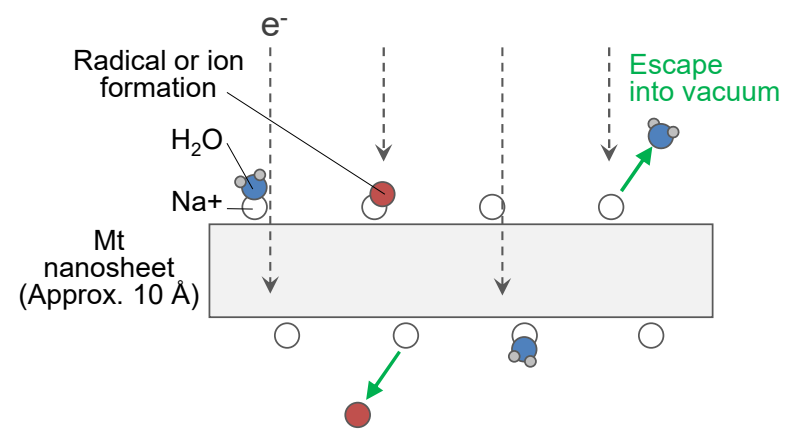

(b) 2L-Mt nanosheet

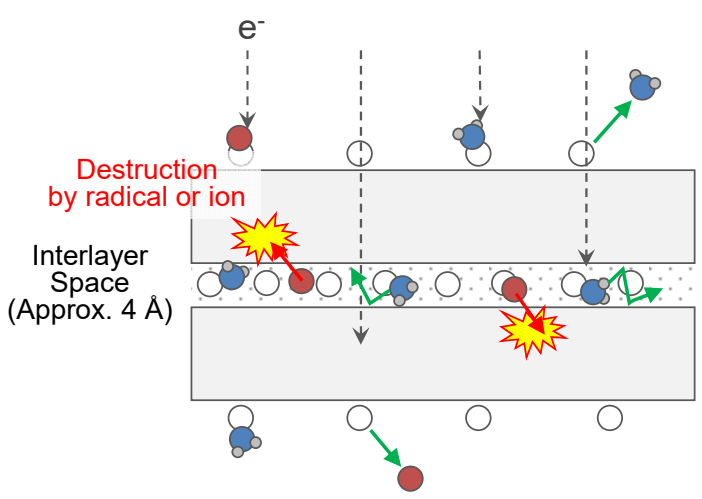

Fig. 4. A plausible schematic illustration for the influence of the existence of the interlayer space on the destruction of (a) $1 \mathrm{~L}$ and (b) $2 \mathrm{~L} \mathrm{Mt}$, representing samples that include interlayer space.

critical doses for $2 \mathrm{~L}$ and $3 \mathrm{~L} \mathrm{Mt}$ were of the same magnitude. According to the results, the absence of the interlayer space governs the difference in stability between $1 \mathrm{~L}$ and multilayered Mt rather than the thickness. All surfaces in the $1 \mathrm{~L} \mathrm{Mt}$ were exposed to vacuum, whereas multilayered $\mathrm{Mt}$ included interlayer spaces (minimum of $4 \AA$ under high vacuum ${ }^{40,41}$ ) as shown in Fig. $4 a$ and b, respectively. Although multiple damage mechanism would proceed simultaneously as described above, the drastic difference of critical dose between $1 \mathrm{~L}$ and $2 \mathrm{~L} \mathrm{Mt}$ can be plausibly explained by the effect of the interlayer space to hold water molecules. Generally, it is practically impossible to completely remove the water from clay minerals. Even after drying by heat or vacuum, certain amount of the water molecules remains in clay minerals due to their high activation energy for dehydration from counter cations under well-dried condition. ${ }^{42-44}$ Kuligiewicz et al. demonstrated that the activation energy of dehydration for water molecules which coordinate the interlayer cations is $90-180 \mathrm{~kJ} / \mathrm{mol}$, while that for bulk water molecules in the interlayer, not directly coordinated to the interlayer cations, is $45-60 \mathrm{~kJ} / \mathrm{mol} .{ }^{45}$ Note that the hydration number of sodium ion is decreased to 0.5-0.6 under well-dried condition. ${ }^{45}$ When these water molecules irradiated by electron beam, excited water molecules, and other molecular species (i.e. $\mathrm{H}^{*}, \mathrm{OH}^{*}, \mathrm{HO}_{2}{ }^{*}, \mathrm{OH}^{-}, \mathrm{H}_{3} \mathrm{O}^{-}, \mathrm{H}_{2}, \mathrm{H}_{2} \mathrm{O}_{2}$ ) are generated, ${ }^{46,47}$ or desorption from the coordinated cations occurs. For the beam damage of zeolite, an aluminosilicate, the lower water content was associated with higher stability during electron microscopy. ${ }^{48,49}$ The generated ions or radical species decrease the strength of the $\mathrm{Si}-\mathrm{O}$ or $\mathrm{Al}-\mathrm{O}$ bonds, followed by a change in the structure. The same mechanism is true for the beam damage in clay minerals. During the observation in the experiment here, water molecules and the generated ions and radical species are hardly released from the interlayer spaces into the vacuum owing to the physical limitation of escape routes to the edges (Fig. 4b); conversely, the same is easy in the monolayered sample, whose surfaces are completely exposed to vacuum, resulting in remarkable stability of $1 \mathrm{~L} \mathrm{Mt}$. The results in Table 3, wherein nanosheets I and II in the $2 \mathrm{~L}$ sample showed the same critical dose, indicate isotropic destruction from the interlayer space.
Furthermore, it is known that $\mathrm{Al}-\mathrm{OH}$ in the octahedral site is relatively unstable, and dehydroxylation in the neighboring Al$\mathrm{OH}$ sites and associated amorphization occurs in clay minerals under high temperature or irradiation. ${ }^{45,50-52}$ The generated ions and radical species can pass through the six-membered rings of the Si-O tetrahedron and attack the $\mathrm{Al}-\mathrm{OH}$ sites to promote dehydroxylation, and thus change the crystal structures. Although we were not able to distinguish the adjacent $\mathrm{Si}$ and $\mathrm{Al}$ atoms in monolayer $\mathrm{Mt}$, ADF-STEM imaging may help monitor the evolution of the dehydroxylation process empirically, which has been previously studied only by thermogravimetry, X-ray diffraction, Fourier-transform infrared spectroscopy, or DFT calculations. ${ }^{37,50,51}$

\section{Conclusions}

Herein, we demonstrated the remarkable stability of freestanding monolayer $\mathrm{Mt}$ against the beam irradiation using electron diffraction technique, while $2 \mathrm{~L}$ and $3 \mathrm{~L} \mathrm{Mt}$ showed similar low stabilities. The results suggested that the stability of $1 \mathrm{~L} \mathrm{Mt} \mathrm{was} \mathrm{owing} \mathrm{to} \mathrm{the} \mathrm{absence} \mathrm{of} \mathrm{interlayer} \mathrm{space,} \mathrm{as} \mathrm{a} \mathrm{site} \mathrm{for}$ hydration, rather than the thinness of the structure. Although complete dehydration of clay minerals was not achieved in the multilayered $\mathrm{Mt}$, the monolayer $\mathrm{Mt}$ with entirely exposed surfaces to the vacuum enables the efficient release of water molecules imparting much less beam damage. Although we focused on $\mathrm{Mt}$ in this work as a good model for experimental demonstration owing to the moderate anionic charge density and the large particle size up to hundreds of nanometers, the results here can be applied for other clay minerals in principle because the hydration is a common phenomenon of clay minerals. The moderate anionic charge density of Mt eases the exfoliations into individual layers in aqueous dispersion, and relatively larger particle sizes $(\sim$ hundreds $\mathrm{nm})$ that is comparable to the illuminated area in TEM have an advantage on avoiding the quick beam damage from edges of layers. Other common clay minerals such as mica (too high charge density) or saponite (sizes of only $\sim$ tens $\mathrm{nm}$ ) are therefore not the good model for this purpose. By using Mt we can thus focus on the 
dependence of electron beam damage on the layer number, i.e. the existence of the interlayer space for the first time. The findings here encourage further investigation using (S)TEM not only for the free-standing monolayer clay minerals but also for a wide variety of nanomaterials, such as the ones that are strongly hydrated in their interlayer spaces or nanopores.

\section{Conflicts of interest}

The authors declare no competing financial interest.

\section{Acknowledgements}

This work has been supported by a Grant-in-Aid for JSPS Fellows DC1 (grant number 18J20894) from Japan Society for the Promotion of Science to I. A. Y.I. acknowledges financial support from JSPS KAKENHI grant number 18K14070, Japan Prize Foundation, The Asahi Glass Foundation, and Foundation for Interaction in Science \& Technology. Partial financial supports by the Cooperative Research Program of "Network Joint Research Center for Materials and Devices" (20201248, 20191100, and 20191201 to T.Y.), Japan, are gratefully acknowledged.

\section{References}

1

F. Carniato, G. Gatti and C. Bisio, An overview of the recent synthesis and functionalization methods of saponite clay, New J. Chem., 2020, 44, 9969-9980.

2 L. A. Utracki, M. Sepehr and E. Boccaleri, Synthetic, layered nanoparticles for polymeric nanocomposites (PNCs), Polym. Adv. Technol., 2007, 18, 1-37. B. S. Kumar, A. Dhakshinamoorthy and K. Pitchumani, K10 montmorillonite clays as environmentally benign catalysts for organic reactions, Catal. Sci. Technol., 2014, 4, 23782396.

4 F. López Arbeloa, V. Martínez Martínez, T. Arbeloa and I. López Arbeloa, Photoresponse and anisotropy of rhodamine dye intercalated in ordered clay layered films, $J$. Photochem. Photobiol. C Photochem. Rev., 2007, 8, 85108.

5 Y. Ishida, Manipulation of supramolecular 2D assembly of functional dyes toward artificial light-harvesting systems, Pure Appl. Chem., 2015, 87, 3-14.

6 H. Sato, K. Takimoto, H. Mori and A. Yamagishi, Stereoselective interactions as manifested by vibrational circular dichroism spectra: The interplay between chiral metal complexes co-adsorbed in a montmorillonite clay, Phys. Chem. Chem. Phys., 2018, 20, 25421-25427. H. Nishina, S. Hoshino, Y. Ohtani, T. Ishida, T. Shimada and S. Takagi, Anisotropic energy transfer in a clay-porphyrin layered system with environment-responsiveness, Phys. Chem. Chem. Phys., 2020, 22, 14261-14267. C. Tan, X. Cao, X. J. Wu, Q. He, J. Yang, X. Zhang, J. Chen, W. Zhao, S. Han, G. H. Nam, M. Sindoro and H. Zhang, Recent
Advances in Ultrathin Two-Dimensional Nanomaterials, Chem. Rev., 2017, 117, 6225-6331.

S. Wang, A. Robertson and J. H. Warner, Atomic structure of defects and dopants in 2D layered transition metal dichalcogenides, Chem. Soc. Rev., 2018, 47, 6764-6794. A. Hashimoto, K. Suenaga, A. Gloter, K. Urita and S. lijima, Direct evidence for atomic defects in graphene layers, Nature, 2004, 430, 870-873.

P. Y. Huang, S. Kurasch, A. Srivastava, V. Skakalova, J. Kotakoski, A. V. Krasheninnikov, R. Hovden, Q. Mao, J. C. Meyer, J. Smet, D. A. Muller and U. Kaiser, Direct imaging of a two-dimensional silica glass on graphene, Nano Lett., 2012, 12, 1081-1086.

P. C. Börner, M. K. Kinyanjui, T. Björkman, T. Lehnert, A. V Krasheninnikov and $U$. Kaiser, Observation of charge density waves in free-standing $1 \mathrm{~T}-\mathrm{TaSe}_{2}$ monolayers by transmission electron microscopy, Appl. Phys. Lett., 2018, 113, 173103.

S. Yamashita, S. Koshiya, T. Nagai, J. Kikkawa, K. Ishizuka and K. Kimoto, Quantitative annular dark-field imaging of single-layer graphene-II: Atomic-resolution image contrast, Microscopy, 2015, 64, 409-418. S. T. Skowron, T. W. Chamberlain, J. Biskupek, U. Kaiser, E. Besley and A. N. Khlobystov, Chemical Reactions of Molecules Promoted and Simultaneously Imaged by the Electron Beam in Transmission Electron Microscopy, Acc. Chem. Res., 2017, 50, 1797-1807.

E. Nakamura, Atomic-Resolution Transmission Electron Microscopic Movies for Study of Organic Molecules, Assemblies, and Reactions: The First 10 Years of Development, Acc. Chem. Res., 2017, 50, 1281-1292. A. Mittelberger, C. Kramberger and J. C. Meyer, Insights into radiation damage from atomic resolution scanning transmission electron microscopy imaging of mono-layer CuPcCl16 films on graphene, Sci. Rep., 2018, 8, 4813. M. A. Gerkman, S. Sinha, J. H. Warner and G. G. D. Han, Direct Imaging of Photoswitching Molecular Conformations Using Individual Metal Atom Markers, ACS Nano, 2019, 13, 87-96.

Y. L. Huang, J. Zheng, Z. Song, D. Chi, A. T. S. Wee and S. Y. Quek, The organic-2D transition metal dichalcogenide heterointerface, Chem. Soc. Rev., 2018, 47, 3241-3264. S. Koshiya, S. Yamashita and K. Kimoto, Microscopic observation of dye molecules for solar cells on a titania surface, Sci. Rep., 2016, 6, 24616.

K. Sohlberg, T. J. Pennycook, W. Zhou and S. J. Pennycook, Insights into the physical chemistry of materials from advances in HAADF-STEM, Phys. Chem. Chem. Phys., 2015, 17, 3982-4006.

T. Imaoka, T. Toyonaga, M. Morita, N. Haruta and K. Yamamoto, Isomerizations of a $\mathrm{Pt}_{4}$ cluster revealed by spatiotemporal microscopic analysis, Chem. Commun., 2019, 55, 4753-4756.

I. Akita, Y. Ishida and T. Yonezawa, Atomic-Scale Imaging of a Free-Standing Monolayer Clay Mineral Nanosheet Using Scanning Transmission Electron Microscopy, J. Phys. Chem. Lett., 2020, 3357-3361. 
T. Okada, Y. Ide and M. Ogawa, Organic-inorganic hybrids based on ultrathin oxide layers: Designed nanostructures for molecular recognition, Chem. Asian J., 2012, 7, 19801992.

24 I. Akita, Y. Ishida and T. Yonezawa, Counting the Layer Number of Free-Standing Montmorillonite Nanosheets Using Annular Dark Field Scanning Transmission Electron Microscopy, Clay Sci., 2019, 23, 41-45.

25 T. Okumura, K. Tamura, E. Fujii, H. Yamada and T. Kogure, Direct observation of cesium at the interlayer region in phlogopite mica, Microscopy, 2014, 63, 65-72.

26 T. Kogure and E. Okunishi, Cs-corrected HAADF-STEM imaging of silicate minerals, J. Electron Microsc. (Tokyo)., 2010, 59, 263-271.

27 T. Kogure and T. Murakami, Direct identification of biotite/vermiculite layers in hydrobiotite using highresolution TEM., Mineral. J., 1996, 18, 131-137.

28 T. Kogure, M. Jige, J. Kameda, A. Yamagishi, R. Miyawaki and R. Kitagawa, Stacking structures in pyrophyllite revealed by high-resolution transmission electron microscopy (HRTEM), Am. Mineral., 2006, 91, 1293-1299. I. D. M. Robertson and R. A. Eggleton, Weathering of granitic muscovite to kaolinite and halloysite and of plagioclase-derived kaolinite to halloysite, Clays Clay Miner., 1991, 39, 113-126.

30 W. J. Roth, B. Gil, W. Makowski, B. Marszalek and P. Eliášová, Layer like porous materials with hierarchical structure, Chem. Soc. Rev, 2016, 45, 3400-3438.

31 T. Takahashi, T. Ohkubo, K. Suzuki and Y. Ikeda, High resolution solid-state NMR studies on dissolution and alteration of Na-montmorillonite under highly alkaline conditions, Microporous Mesoporous Mater., 2007, 106, 284-297.

32 H. Seyama and M. Soma, X-Ray Photoelectron and Auger Electron Spectroscopic Study of Mg-Montmorillonite, Chem. Lett., 1981, 10, 1009-1012.

33 G. Brown, Ed., The X-ray identification and crystal structures of clay minerals, Mineralogical Society, Clay Minerals Group, London, 1961.

34 J. Środoń, V. A. Drits, D. K. Mccarty, J. C. C. Hsieh and D. D. Eberl, Quantitative X-Ray Diffraction Analysis of ClayBearing Rocks from Random Preparations, Clays Clay Miner., 2001, 49, 514-528.

35 C. I. Rich, Determination of (060) Reflections of Clay Minerals by Means of Counter Type X.Ray Diffraction Instruments, Am. Mineral., 1957, 42, 569-570. $\mathrm{H}$. Seiler, Secondary electron emission in the scanning electron microscope, J. Appl. Phys., 1983, 54, R1.

37 N. Jiang, Electron beam damage in oxides: A review, Reports Prog. Phys., 2015, 79, 016501.

38 R. F. Egerton, Radiation damage to organic and inorganic specimens in the TEM, Micron, 2019, 119, 72-87. S. T. Skowron, S. L. Roberts, A. N. Khlobystov and E. Besley, The effects of encapsulation on damage to molecules by electron radiation, Micron, 2019, 120, 96-103.

Properties of Dioctahedral Smectites, Clays Clay Miner., 1992, 40, 103-113.

E. Ferrage, B. Lanson, B. A. Sakharov and V. A. Drits, Investigation of smectite hydration properties by modeling experimental X-ray diffraction patterns: Part I:

Montmorillonite hydration properties, Am. Mineral., 2005, 90, 1358-1374.

R. W. Mooney, A. G. Keenan and L. A. Wood, Adsorption of Water Vapor by Montmorillonite. II. Effect of Exchangeable Ions and Lattice Swelling as Measured by X-Ray Diffraction, J. Am. Chem. Soc., 1952, 74, 1371-1374.

J. Środoń and K. D. McCarty, Surface Area and Layer Charge of Smectite from CEC and EGME/ $\mathrm{H}_{2} \mathrm{O}$-Retention Measurements, Clays Clay Miner., 2008, 56, 155-174.

R. A. Schoonheydt and C. T. Johnston, in Layered Mineral Structures and their Application in Advanced Technologies, eds. M. F. Brigatti and A. Mottana, Mineralogical Society of Great Britain and Ireland, 2011, vol. 11, pp. 337-373. A. Kuligiewicz and A. Derkowski, Tightly bound water in smectites, Am. Mineral., 2017, 102, 1073-1090.

A. J. Swallow, Radiation chemistry; an introduction, Longman, London, 1973.

T. J. Woehl, K. L. Jungjohann, J. E. Evans, I. Arslan, W. D. Ristenpart and N. D. Browning, Experimental procedures to mitigate electron beam induced artifacts during in situ fluid imaging of nanomaterials, Ultramicroscopy, 2013, 127, 5363.

L. A. Bursill, E. A. Lodge and J. M. Thomast, Zeolitic structures as revealed by high-resolution electron microscopy, Nature, 1980, 286, 111-113.

R. Csencsits and R. Gronsky, Damage of zeolite $Y$ in the TEM and its effects on TEM images, Ultramicroscopy, 1987, 23, 421-431.

T. Allard, E. Balan, G. Calas, C. Fourdrin, E. Morichon and S. Sorieul, Radiation-induced defects in clay minerals: A review, Nucl. Instrum. Meth. B, 2012, 277, 112-120.

C. Fourdrin, T. Allard, I. Monnet, N. Menguy, M. Benedetti and G. Calas, Effect of Radiation-Induced Amorphization on Smectite Dissolution, Environ. Sci. Technol., 2010, 44, 2509-2514.

T. Kogure, Imaging of dioctahedral 2:1 layers by highresolution transmission electron microscopy (HRTEM): Possibility of recording the dehydroxylate, Am. Mineral., 2007, 92, 1368-1373. 